\title{
Análisis de la interrelación crecimiento-rentabilidad en Brasil
}

\section{Analysis of the relationship business growth and profitability in Brazil}

\section{J. Daza Izquierdo}

Universidad de Extremadura, Departamento de Economía Financiera y Contabilidad, miembro del Grupo de Investigación en Mercados y Activos Financieros (GIMAF), Facultad de Ciencias Económicas y Empresariales, Av. Elvas s/n 06071, Badajoz, España, juliodaza@unex.es

\begin{abstract}
Resumen
Actualmente las economías emergentes, y en especial Brasil, son las que están proporcionando mayores oportunidades de negocio a nivel mundial, al alcanzar altas tasas de crecimiento y rentabilidad empresarial. En este contexto, el objetivo del presente estudio consiste en analizar la relación entre crecimiento y rentabilidad empresarial para un panel de 1246 empresas que tienen su actividad en Brasil durante los años 2002 a 2012. La metodología de estudio consiste en un análisis estático por mínimos cuadrados con efectos fijos y un análisis dinámico por el método generalizado de los momentos. Los resultados obtenidos nos indican que existe una clara influencia de la rentabilidad sobre el crecimiento empresarial, pero no al contrario, y que el signo de esta relación difiere si distinguimos según la propiedad y el control de las empresas. Estos resultados pueden ser utilizados por empresarios que deseen realizar su actividad productiva en Brasil o por las autoridades públicas para llevar a cabo políticas de desarrollo económico y social.
\end{abstract}

Palabras clave: Crecimiento y rentabilidad empresarial, mercados emergentes, datos de panel.

\begin{abstract}
Currently emerging economies, especially Brazil, are providing more business opportunities globally in terms of higher growth rates and corporate profitability. In this context, the aim of this paper is to analyze the relationship between firm growth and profitability for a panel of 1246 firms with their activity in Brazil during the 2002-2012 period. The methodology we employ consists of a static test using least squared with fixed effects, and a dynamic test using the generalized method of moments. Our results indicate the existence of a clear influence of profitability on firm growth, but not vice versa, and that the sign of this relationship differs if we distinguish according to ownership and control of companies. These results can be used by managers who wish to make their business activities in Brazil or by the public authorities to pursue economic and social development policies.
\end{abstract}

Keywords: Firm growth and profitability, emerging markets, panel data.

\section{Introducción}

Existe una amplia evidencia empírica para dar respuesta a la eterna pregunta de por qué unas empresas crecen más que otras (Sutton, 1997; Suárez Suárez, 2014). Esta cuestión ha ido dirigida a empresas industriales y de servicios situadas en los países más desarrollados, ya que reportaban altas rentabilidades a sus inversores y eran más seguras. Pero actualmente, debido a la coyuntura económica, los países más desarrollados han visto mermadas sus rentabilidades y crecimiento.

Los inversores europeos y norteamericanos tienen dificultades de mantener los niveles de rentabilidad de sus inversiones en los países más desarrollados debido a la coyuntura económica internacional. Por ello, que intenten mantener el nivel de sus rentabilidades dirigiendo sus inversiones a los países emergentes, que según señalan Orgaz, Molina y Carrasco (2011) han crecido a un ritmo superior que las economías desarrolladas en la última década. Según indica López Vicente (2013) destaca el crecimiento de la economía brasileña sobre la de otros países emergentes. Además, Serena Garralda (2011) y Arnold y Jalles (2014) destacan que Brasil afrontó positivamente la crisis financiera y pudo adoptar medidas contracíclicas, gracias a las reformas estructurales llevadas a cabo en la década de los noventa

La ley del Efecto proporcional y el estudio de la persistencia de la rentabilidad son los pilares en los que se apoya el estudio de la interrelación entre crecimiento y rentabilidad empresarial. Tradicionalmente se ha estudiado el crecimiento empresarial desde un punto de vista empírico basado en el comportamiento estocástico del crecimiento defendido en la ley del Efecto proporcional. La ventaja de dicha ley es la de aproximar el comportamiento del crecimiento empresarial a una distribución log-normal conocida, descrita por la distribución del tamaño. De este modo resulta más sencillo el estudio del crecimiento de la empresa, debido a la capacidad para incluir más variedad de factores que afectan a la misma, dependiendo del país, el sector y el periodo en el cual son tomados los datos.

El principal objetivo del trabajo es estudiar la interacción entre el crecimiento y la rentabilidad empresarial y sus factores explicativos, usando una base de datos de las 1246 empresas que tienen actividad en Brasil, siendo el periodo de observación de la muestra de 2002 a 2012. Además se contrasta la ley del efecto proporcional y la persistencia de la rentabilidad. Las empresas son clasificadas en función de sus características de propiedad y control, destacando de este modo la influencia y resultados de las empresas extranjeras. Los resultados obtenidos aportan evidencia empírica en un país emergente que pueden ser utilizados por empresas e inversores que deseen conocer y desarrollar actividades productivas en Brasil o por las autoridades para desarrollar políticas regionales de desarrollo económico y social.

El resto del artículo es organizado como se describe a continuación. En el apartado segundo se muestra una revisión de la literatura del estudio del crecimiento. En el tercer apartado se presenta la metodología utilizada. En el cuarto apartado se describe la base de datos empleada. En el quinto aparatado se presentan los resultados empíricos obtenidos. Por último, se presenta un sexto apartado que contiene las conclusiones derivadas del conjunto del trabajo. 


\section{Marco teórico}

El estudio del crecimiento empresarial tiene por objeto conocer por qué unas empresas crecen más que otras, cuál es el motivo de su éxito y los factores que influyen en su crecimiento. A pesar de los estudios realizados, todavía no existe el desarrollo de un decálogo para alcanzar altas tasas de crecimiento y todavía menos las claves para alcanzar el éxito empresarial. Por esta razón, se considera que el crecimiento empresarial es el resultado de la combinación de factores aleatorios tal y como defiende la ley del Efecto proporcional.

La ventaja de la ley del Efecto proporcional es facilitar la incorporación de nuevos factores explicativos para el estudio del crecimiento empresarial. Dicha ley relaciona el crecimiento empresarial con la distribución del tamaño empresarial mediante una función conocida log-normal. Adicionalmente, el estudio y contraste de la ley del Efecto proporcional es interesante en las economías emergentes, especialmente Brasil. En primer lugar, porque las economías emergentes son las economías de mayor desarrollo económico y empresarial a nivel mundial y, en segundo lugar, porque Brasil es uno de los principales destinos de la inversión extranjera directa.

Según los resultados del contrastar la relación entre las tasas de crecimiento y tamaño empresarial, Wagner (1992) propone diferentes políticas regionales para cada uno de los tres posibles escenarios resultantes. A continuación se describen cada una de las combinaciones entre crecimiento y tamaño y el enfoque que deben tomar las decisiones de políticas regionales en cada caso.

Si las empresas pequeñas crecen más deprisa que las grandes, las políticas regionales deberían ir dirigidas a fomentar el nacimiento y desarrollo de las pequeñas empresas, como por ejemplo mediante subvenciones, ofreciendo asesoramiento a bajo coste y otro tipo de actividades de formación, ya que así se fomenta la dinamización en el empleo y, por tanto, la creación de empleo neto.

Otro caso puede ser que de la relación existente entre tamaño y crecimiento se llegue a la conclusión de que las empresas de mayor tamaño crecen más deprisa. De este modo las políticas regionales deberían ir dirigidas a favorecer la localización de este tipo de empresas en la región, mediante incentivos que mantengan las ya existentes y que atraigan otras foráneas.

Si el tamaño y el crecimiento son independientes indicaría que las políticas regionales enfocadas a las empresas o a fomentar el empleo no deben ser orientadas en función del tamaño empresarial. Por lo tanto, se han de identificar cuáles son los factores que influyen en el crecimiento y actuar sobre ellos.

A consecuencia de este último caso, Wagner (1992) resalta la influencia de las características ambientales y del azar sobre la empresa, y de forma particular, en el proceso de crecimiento empresarial y en la necesidad de poder contemplarlas en los modelos. Además, la separación entre propiedad y control y la importancia de las decisiones de los directivos implica, como destaca Pettinger (2013), que alcanzar los objetivos empresariales se vean condicionado por las necesidades humanas.

Dentro de la literatura actual acerca de la ley del Efecto proporcional, el crecimiento empresarial no solo se ha ido estudiando en su relación con el tamaño sino también en relación con la rentabilidad, siendo esta una de las relaciones que más influencia e interés está teniendo en la actualidad. Entre los autores más relevantes en el estudio de la relación entre estas dos magnitudes empresariales, destacan Goddard, Molyneux y Wilson (2004), Cowling (2004), Coad (2007, 2009), Jang y Park (2011) y Lee (2014) no llegando a iguales resultados.

Estos trabajos analizan el crecimiento empresarial contrastando si la tasa de crecimiento empresarial no está relacionada con el tamaño empresarial ni con la tasa de crecimiento anterior. Si estas relaciones no se cumplen, entonces el crecimiento empresarial sigue un proceso aleatorio en el que la varianza puede incrementarse indefinidamente. Pero esta hipótesis implicaría que no existen límites en el mercado a largo plazo, sin embargo, si la tasa de crecimiento estuviera relacionada inversamente con el tamaño empresarial, el crecimiento empresarial tendería a converger en el largo plazo.

De este modo, y siguiendo las pautas de persistencia de rentabilidad (persistence of profitability, POP) se asume que la rentabilidad empresarial tiende a converger en un punto provocado por la competencia de los mercados, es decir, que en condiciones en las que la entrada y salida de empresas de los mercados fuera libre, las rentabilidades anormales desaparecerían y la rentabilidad de las empresas del mercado tenderían a concentrarse a largo plazo.

Según las teorías gerenciales el crecimiento y la rentabilidad deben estar relacionados debido a que son los principales objetivos empresariales. Esto no está totalmente asegurado y menos aún que tipo de relación existe entre ellas, tampoco si la influencia va en una sola dirección o en ambos sentidos, con que signo y en qué proporción. Algunos trabajos recientes como el de Jang y Park (2011) realizado sobre empresas de los Estados Unidos llegan a la conclusión de que la tasa de rentabilidad tiene un efecto positivo en el crecimiento empresarial, pero que el crecimiento empresarial tienen un efecto negativo sobre la rentabilidad. Por el contrario, Lee (2014) encuentra relaciones opuestas para empresas cuando se trata de país no desarrollado, señalando el efecto país como factor explicativo.

De este modo, Jang y Park (2011) destacan tres tipos de comportamiento de la rentabilidad empresarial en función del tiempo y de su relación con el crecimiento empresarial. En primer lugar, las tasas de rentabilidad tienden a converger en el largo plazo, cumpliéndose la persistencia de la rentabilidad. En segundo lugar, las altas tasas de rentabilidad tienen un efecto positivo sobre el crecimiento empresarial, hasta que la tasa de rentabilidad sea próxima a cero. Y en tercer lugar, destaca la influencia negativa del crecimiento empresarial sobre las tasa de rentabilidad, justificado por la ejecución masiva de proyectos aunque estos no sean rentables. 
En el caso de que las rentabilidades anormales permanezcan en el tiempo, sugiere que las empresas poseen algún tipo de información especial o alguna ventaja de su posición competitiva. De este modo, la persistencia de las tasas de rentabilidad combinada en ocasiones con el crecimiento empresarial, puede estar provocado por factores internos de la empresa como puede ser la eficiencia y el saber hacer de los directivos.

Por último, cabe señalar que tradicionalmente los resultados variaban en gran medida por problemas en la selección de la muestra y por el uso no adecuado de las herramientas econométricas. En este aspecto, el presente trabajo utiliza datos de panel y aplica las herramientas necesarias para solventar los posibles problemas de heterogeneidad inobservable y endogeneidad.

\section{Metodología}

En este apartado se presenta la metodología que es utilizada para analizar la interrelación entre crecimiento y rentabilidad. La metodología utilizada se basa en la estructura de datos de panel que permite tener en cuenta la heterogeneidad inobservable que existe entre las distintas empresas permitiendo además aumentar el número de observaciones, los grados de libertad de los modelos y por tanto la consistencia de los resultados (García-Ramos \& García-Olalla, 2011).

Existen principalmente dos problemas econométricos en la metodología de datos de panel, la heterogeneidad inobservable y el problema de endogeneidad. En primer lugar, el problema de heterogeneidad inobservable se soluciona incluyendo en el modelo las variables de efectos específicos de cada empresa, que formará parte a su vez del término error y será estimado mediante Mínimos Cuadrados Ordinarios (MCO) de Efectos Fijos. En segundo lugar, el problema de endogeneidad se afronta mediante diversos métodos de variables instrumentales. Para dar

solución a estos problemas se hace uso del Método Generalizado de los Momentos (GMM), ya que este método es un caso particular en la aplicación de variables instrumentales cuando se utilizan las variables explicativas como instrumentos.

Los estimadores GMM son el resultado de una amplia tendencia histórica en la práctica econométrica enfocada hacia estimadores que hacen menos suposiciones sobre el proceso de generación de datos subyacentes, ya que utiliza técnicas más complejas para aislar la información útil, según señala Roodman (2009). Además señala que los estimadores GMM están diseñados para analizar los datos de panel y procesos dinámicos.

Por lo tanto, se realiza un análisis estático y un análisis dinámico para comprobar la interrelación crecimientorentabilidad, se contrasta la ley del Efecto proporcional y además se comprueba la persistencia del crecimiento y de la rentabilidad. Para obtener estimadores consistentes y robustos en los modelos estáticos y dinámicos se utilizarán los métodos de Efecto Fijos y Método Generalizado de los Momentos respectivamente.

En primer lugar, se realiza un análisis estático del crecimiento y de la rentabilidad para comprobar la relación entre crecimiento y rentabilidad. La estimación de los modelos estáticos de datos de panel debe realizarse mediante el método de MCO de Efectos Fijos, con el objetivo de controlar el problema de heterogeneidad inobservable de las empresas. De esta forma se precisa si existen cambios en la variable dependiente como causa de los cambios producidos por las propias empresas con el paso de los años, o por el contrario, se debe a Efectos Aleatorios. La estimación del modelo se realiza por el método de Mínimos Cuadrados Ordinarios contrastando la existencia de Efectos Fijos o Efectos Aleatorios mediante el test de Hausman. El modelo es expresado del siguiente modo:

$$
\begin{aligned}
& \operatorname{Crec}_{i, t}=c+\beta_{1} \operatorname{Re}_{i, t-1}+\beta_{2} \operatorname{Tam}_{i, t}+\beta_{3} \operatorname{End}_{i, t}+\beta_{4} \operatorname{Liq}_{i, t}+D A \tilde{n} o_{i, t}+\varepsilon_{i, t} \\
& \operatorname{Re} n t_{i, t}=c+\beta_{1} \operatorname{Crec}_{i, t-1}+\beta_{2} \operatorname{Tam}_{i, t}+\beta_{3} \text { End }_{i, t}+\beta_{4} \operatorname{Liq}_{i, t}+D A \tilde{n} o_{i, t}+\varepsilon_{i, t}
\end{aligned}
$$

donde $\operatorname{Crec}_{i, \mathrm{t}}$ y Rent $_{i, \mathrm{t}}$ representa el crecimiento y la rentabilidad del banco $i$ en el momento $t$ respectivamente, que viene explicado por las variables endógenas retardadas del crecimiento y la rentabilidad un periodo, además de un conjunto de variables de control que representan el tamaño empresarial, el endeudamiento, la tasa de liquidez y las variables dicotómicas temporales. Por último, $c$ y $\beta_{i}$ son los parámetros a estimar y $\varepsilon_{i, t}$ el término de error.

Los modelos estáticos propuestos para estudiar la relación entre crecimiento y rentabilidad están compuestos de modo que el crecimiento sea explicado en función de la rentabilidad y viceversa, estando las variables explicativas retardadas un periodo para evitar problemas de endogeneidad. También se incorporan en los modelos variables de control como el tamaño empresarial, para contrastar la ley del Efecto proporcional, y el endeudamiento y la liquidez, así como las variables dicotómicas temporales de año.
La medida del crecimiento empresarial ha sido calculada tomando la diferencia de logaritmos del tamaño empresarial entre dos periodos consecutivos, siguiendo a Coad, Rao y Tamgni (2011). El tamaño empresarial, $\operatorname{Tam}_{i, t}$ es medido como el logaritmo natural de la cifra de ventas. La medida de rentabilidad utilizada es la rentabilidad de los recursos propios como en el trabajo de Goddard et al. (2004).

El estudio del crecimiento empresarial no se limita al contraste de la ley del Efecto proporcional, si no que existen variables financieras que explican el comportamiento del crecimiento empresarial. Por ello, siguiendo a Goddard et al. (2004), Coad (2007) y Jang y Park (2011) se ha incorporado como variable explicativa en el modelo la rentabilidad, expresado como Rent ${ }_{i, t}$. Siguiendo a Goddard et al. (2004), se utiliza rentabilidad de los recursos propios de la empresa.

El endeudamiento se ha incorporado como variable de control, mediante Endi,t siguiendo los trabajos presentados por Oliveira y Fortunato (2006) y Jang y Park (2011), de este modo, el endeudamiento es calculado por el cociente entre 
las deudas totales y las exigencias a largo plazo. Billett, King y Mauer (2007) empíricamente evidenció una relación negativa entre la tasa de crecimiento y aquellas empresas más endeudadas, por ello, se defiende que la influencia del apalancamiento en el crecimiento puede ser negativa.

Siguiendo a Oliveira y Fortunato $(2006,2008)$, otra de las variables de control incluidas en el modelo es la liquidez, expresada como $L i q_{i, t}$, y es definida por la relación entre el activo circulante y el pasivo circulante. Broome, Robinson y Moore (2009) obtiene un efecto positivo entre liquidez y crecimiento, pero obtiene una relación inversa para las empresas con exceso de liquidez.
Por otra parte, se incorpora el factor propiedad de las empresas como condicionante para evaluar la diferencias de comportamiento y de interrelación entre crecimiento y rentabilidad, siguiendo el trabajo de Hsieh y Lee (2010). De este modo, desglosamos la muestra según sean las empresas de propiedad privada o pública y de control brasileño o extranjero. De este modo, tenemos tres submuestras, las estatales o de propiedad pública y, dentro de las de propiedad privada, brasileñas y extranjeras.

En segundo lugar, y siguiendo con un análisis lineal, se presentan los modelos dinámicos para examinar la relación crecimiento-rentabilidad, donde se incluyen en las ecuaciones 1 y 2 las variables dependientes retardadas, expresando los modelos dinámicos del siguiente modo:

$$
\begin{aligned}
& \text { Crec }_{i, t}=c+\beta_{1} \text { Crec }_{i, t-1}+\beta_{2} \operatorname{Re}_{i, t-1}+\beta_{3} \operatorname{Tam}_{i, t-1}+\beta_{4} \operatorname{End}_{i, t}+\beta_{5} \operatorname{Liq}_{i, t}+D A \tilde{n} o_{i, t}+\varepsilon_{i, t} \\
& \operatorname{Re} n t_{i, t}=c+\beta_{1} \operatorname{Re} n t_{i, t-1}+\beta_{2} \operatorname{Crec}_{i, t-1}+\beta_{3} \operatorname{Tam}_{i, t-1}+\beta_{4} \operatorname{End}_{i, t}+\beta_{5} \operatorname{Liq}_{i, t}+D A \tilde{n} o_{i, t}+\varepsilon_{i, t}
\end{aligned}
$$

En el caso de los modelos dinámicos la estimación mediante Mínimos Cuadrados Ordinarios tanto con Efectos Fijos como Aleatorios proporcionan resultados sesgados, por lo que se propone la utilización de variables instrumentales. El método elegido para obtener estimaciones robustas y eficientes es el Método Generalizado de los Momentos en diferencias (Arellano y Bond, 1991) que se trata de un método particular de variables instrumentales. En el caso de que la variable dependiente sea persistente se utiliza el estimador de sistemas del Método Generalizado de los Momentos propuesto por Blundel y Bond (1998).

Los instrumentos utilizados para solucionar el problema de endogeneidad deberán estar correlacionados con el término error, pero deberán tener la capacidad de aportar la información sobre la variable explicativa. Coincidiendo con Coad (2007) y Lee (2014) se ha elegido la variable dependiente retardada solo dos periodos para usarla como instrumentos para la estimación del modelo, debido a que el uso de un mayor número de retardos no proporcionan información económica relevante.

Por último, para comprobar significación global del modelo se observa el test de la F. Además se realiza el test de Wald para comprobar por separado la significación conjunta de los coeficientes de las variables explicativas y del conjunto de coeficientes de las variables dicotómicas temporales. Para comprobar la validez de los instrumentos se usa el test de Sargan de sobreidentificación de los instrumentos. Se realiza el test estadístico de Arellano y Bond (1991) para comprobar la ausencia de autocorrelación de segundo orden $\mathrm{m}_{2}$.

\section{Base de datos}

La muestra utilizada se compone de un total de 1246 empresas obtenidas de la base de datos Exame durante el periodo comprendido entre 2002 y 2012. El conjunto de las empresas obtenidas se caracterizan por ser las mayores empresas con actividad en Brasil, clasificando las empresas según su propiedad y control, diferenciando en primer lugar entre propiedad estatal o privada, y éstas últimas a su vez en función de si son controladas por capital brasileño o extranjero. En la Tabla 1 se describe la muestra clasificada en función de la propiedad y el control y la evolución del valor medio del crecimiento para cada una de las submuestras durante el periodo analizado.

\begin{tabular}{|c|c|c|c|c|}
\hline \multicolumn{5}{|c|}{ Panel A. Características según el tipo de empresa } \\
\hline Tipo de Empresa & Número & Tamaño & Crecimiento & Rentabilidad \\
\hline Estatal & 71 & 7,98 & $-0,15$ & 5,36 \\
\hline Brasileño & 878 & 7,98 & 0,14 & 8,64 \\
\hline Extranjero & 197 & 7,82 & 1,14 & 5,35 \\
\hline Total & 1246 & 7,93 & 0,43 & 7,64 \\
\hline \multicolumn{5}{|c|}{ Panel B. Evolución del crecimiento empresarial } \\
\hline Año & Estatal & Brasileño & Extranjero & Total general \\
\hline 2003 & 33,12 & 15,55 & $-9,26$ & 6,89 \\
\hline 2004 & 14,06 & $-19,84$ & $-7,47$ & $-11,52$ \\
\hline 2005 & $-15,13$ & 2,15 & 14,10 & 5,45 \\
\hline 2006 & $-25,54$ & 4,15 & $-7,55$ & $-3,46$ \\
\hline 2007 & $-10,28$ & $-9,68$ & 7,80 & $-2,69$ \\
\hline 2008 & 0,88 & 1,55 & $-2,03$ & 0,03 \\
\hline 2009 & 43,43 & 2,27 & $-0,56$ & 4,86 \\
\hline 2010 & $-19,06$ & $-1,63$ & $-12,61$ & $-5,59$ \\
\hline 2011 & $-1,10$ & $-4,62$ & 10,05 & $-0,80$ \\
\hline 2012 & $-3,17$ & 4,72 & 0,13 & 3,14 \\
\hline
\end{tabular}

Tabla 1 - Descripción y evolución de la muestra según propiedad y control

La tabla presenta la descripción de muestra clasificada en función de la propiedad y el control, resultando tres tipos de empresas: Estatal (Pública-brasileña), Brasileño (Privada-brasileño) y Extranjero (Privada-extranjero). En el Panel A se detalla el número de empresas y el promedio medio del tamaño, crecimiento y rentabilidad En el Panel B se describe la evolución del promedio de crecimiento empresarial para cada tipo de entidad. 
En el Panel A de la Tabla 1 se desglosan las 1246 empresas utilizadas en 71 empresas Estatales, 878 Brasileñas y 297 Extranjeras. En el Panel B de la Tabla 1 se observa la evolución del crecimiento empresarial en función de la propiedad para cada año. Destacando el decrecimiento de las empresas extranjeras en los años 2008, 2009 y 2010.

Las variables recogidas en la muestra son la cifra de ventas, rentabilidad, endeudamiento y liquidez. La cifra de venta ha sido utilizada como medida del tamaño empresarial, que en este trabajo es estimado como el logaritmo neperiano de las cifra de ventas. Dicha medida de crecimiento ha sido utilizada por diversos autores como Jang y Park (2011) y García-Majón y Romero-Merino (2012).

Los estadísticos descriptivos de las muestra se presentan en la Tabla 2. El análisis realizado muestra estadísticos tales como la media, mediana, desviación típica, así como valores máximos y mínimos. También se muestran los resultados del test de estacionariedad realizado siguiendo los contrastes de Dickey-Fuller Aumentado (ADF) y Philips Perron (PP), utilizando los estadísticos Fisher-Chicuadrado. Este test es necesario, porque para realizar la estimación de modelos dinámicos de datos de panel es necesario que las variables utilizadas sean estacionarias.

Tabla 2 - Estadísticos descriptivos y matriz de correlaciones. Muestra total.

\begin{tabular}{|c|c|c|c|c|c|}
\hline \multicolumn{6}{|c|}{ Panel A. Estadísticos descriptivos } \\
\hline & Crecimiento & Rentabilidad & Tamaño & Endeudamiento & Liquidez \\
\hline Media & 0,97 & 10,70 & 7,93 & 24,13 & 1,21 \\
\hline Mediana & 0,56 & 10,50 & 8,16 & 20,50 & 1,00 \\
\hline Máximo & 319,87 & 99,80 & 8,90 & 232,00 & 69,30 \\
\hline Mínimo & $-323,08$ & $-99,40$ & 0,00 & 0,00 & $-21,70$ \\
\hline Desv. Típica & 58,08 & 18,99 & 0,93 & 19,63 & 1,55 \\
\hline ADF & $2548,13^{* * *}$ & $2722,05^{* * *}$ & $2915,98^{* * *}$ & $2235,65^{* * *}$ & $1711,55^{* * *}$ \\
\hline PP & $4160,86^{* * *}$ & $3075,44^{* * *}$ & $3292,79^{* * *}$ & $2678,05^{* * *}$ & $1876,46^{* * *}$ \\
\hline \multicolumn{6}{|c|}{ Panel B. Matriz de correlaciones } \\
\hline & Crecimiento & Rentabilidad & Tamaño & Endeudamiento & Liquidez \\
\hline Crecimiento & 1,00 & & & & \\
\hline Rentabilidad & $-0,00$ & 1,00 & & & \\
\hline Tamaño & $0,29 * *$ & $-0,01$ & 1,00 & & \\
\hline Endeudamiento & 0,01 & $-0,06^{* *}$ & 0,00 & 1,00 & \\
\hline Liquidez & 0,00 & $-0,00$ & 0,01 & $-0,25^{* *}$ & 1,00 \\
\hline
\end{tabular}

Los estadísticos ADF y PP mostrados en el Panel A de la Tabla 2 muestran que todas las variables son estacionarias. Se observa que el tamaño medio de las empresas, medido por el neperiano de las ventas, es de $7,93 \%$ y la mediana es de $8,16 \%$ debido a la presencia de empresas de gran tamaño en la muestra. La tasa media de crecimiento sobre las ventas es de $0,97 \%$ y la mediana de $0,56 \%$, siendo la desviación típica de 58,08. La rentabilidad media de las empresas de Brasil es de 10,70\% y la mediana de 10,50\%. Las empresas brasileñas están endeudadas de media en un $24,13 \%$, su tasa de liquidez es del 1,21\% existiendo diferencias significativas de concentración de unos sectores a otros. En el Panel B de la Tabla 2, se muestra la matriz de correlaciones entre las variables. Se observa que las variables tamaño y crecimiento empresarial están correlacionadas positivamente al contrario que las variables endeudamiento y liquidez.

\section{Resultados}

En este apartado se presentan los resultados del trabajo mostrando los resultados obtenidos de los modelos estáticos y dinámicos planteados mediante MCO con Efectos Fijos o Aleatorios y GMM respectivamente, desglosando los resultados de la muestra general en función de la propiedad y el control de las empresas.

En primer lugar, los resultados obtenidos procedentes del análisis estático de la interrelación crecimiento rentabilidad se muestran en la Tabla 3. En primer lugar, el análisis del crecimiento empresarial ha sido realizado mediante MCO con presencia de Efectos Fijos para la muestra total y para las empresas privadas brasileñas y extranjeras. De este modo, se han encontrado evidencias de influencia de la rentabilidad sobre el crecimiento en las empresas privadas, siendo positivas en las brasileñas y negativa en las extranjeras. Por lo tanto, la propiedad y el control condicionan la relación de la rentabilidad sobre el crecimiento. Además también se observa de forma generalizada en todas las muestras que el tamaño empresarial influye positivamente sobre el crecimiento, de manera que se rechaza la ley del Efecto proporcional. 
Tabla 3 - Análisis Estático de la Interrelación Crecimiento-Rentabilidad

\begin{tabular}{|c|c|c|c|c|c|c|c|c|c|}
\hline \multicolumn{5}{|c|}{ Análisis Estático del Crecimiento/Efectos Fijos o Aleatorios } & \multicolumn{5}{|c|}{ Análisis Estático de la Rentabilidad/ Efectos Fijos o Aleatorios } \\
\hline & Total & Estatal & Brasileño & Extranjero & & Total & Estatal & Brasileño & Extranjero \\
\hline \multirow[t]{2}{*}{ Rent $(-1)$} & 0,003 & $-0,014$ & 0,033* & $-0,034^{*}$ & Crec (-1) & $-0,000$ & 0,008 & 0,004 & 0,000 \\
\hline & $(0,25)$ & $(-0,25)$ & $(1,89)$ & $(-1,74)$ & & $(-0,01)$ & $(1,39)$ & $(0,15)$ & $(0,13)$ \\
\hline \multirow[t]{2}{*}{ Tamaño } & $35,795^{* * *}$ & $5,614^{*}$ & $35,850^{* * *}$ & $43,353^{* * *}$ & Tamaño & $-0,482^{*}$ & $-0,270$ & $-0,260$ & $-1,009$ \\
\hline & $(23,99)$ & $(1,95)$ & $(20,09)$ & $(13,12)$ & & $(-1,66)$ & $(-0,32)$ & $(-0,82)$ & $(-1,57)$ \\
\hline \multirow[t]{2}{*}{ End } & $0,224^{* *}$ & 0,030 & 0,339 ** & 0,049 & End & $-0,171^{* * *}$ & $-0,109$ & $-0,139 * * *$ & $-0,182^{* * *}$ \\
\hline & $(2,22)$ & $(0,20)$ & $(2,66)$ & $(0,24)$ & & $(-6,18)$ & $(-1,18)$ & $(-5,62)$ & $(-3,50)$ \\
\hline \multirow[t]{2}{*}{ Liq } & 0,597 & $-0,275$ & 0,304 & 0,671 & Liq & 0,047 & $-1,347$ & $-0,055$ & 0,149 \\
\hline & $(0,67)$ & $(-0,08)$ & $(0,27)$ & $(0,42)$ & & $(0,17)$ & $(-0,72)$ & $(-0,22)$ & $(0,38)$ \\
\hline $\mathrm{R}^{2}$ & 0,1608 & 0,0552 & 0,1709 & 0,2078 & $\mathrm{R}^{2}$ & 0,0575 & 0,0122 & 0,0198 & 0,0235 \\
\hline Wald test & $43,94^{* * *}$ & 15,20 & $31,74^{* * *}$ & $14,04^{* * *}$ & Wald test & $12,22^{* * *}$ & 0,89 & $32,59 * * *$ & $14,98^{* * *}$ \\
\hline $\begin{array}{c}\text { Test } \\
\text { Hausman }\end{array}$ & $274,59^{* * *}$ & 9,90 & $196,46^{* * *}$ & $138,00^{* * *}$ & $\begin{array}{c}\text { Test } \\
\text { Hausman }\end{array}$ & $84,38^{* * *}$ & 1,27 & $9,21^{*}$ & 0,1382 \\
\hline
\end{tabular}

En la tabla se recogen los resultados de la estimación de los modelos estáticos de crecimiento y rentabilidad estimados mediante Efecto Fijos o Aleatorios. Crecimiento (-1) y Rentabilidad (-1) son las variables retardadas un periodo. Se incluyen las variables de control Tamaño, Endeudamiento y Liquidez. La estimación de Efectos Fijos o Aleatorios se incorpora la bondad del ajuste expresado en $\mathrm{R}^{2}$, la significación conjunta de los coeficientes es expresada mediante el test de Wald. También se muestra el test de Hausman para testar la existencia o no de Efectos Fijos, tratándose de una estimación de Efectos Fijos cuando el resultado del test es significativo al 5\%, siendo estimado mediante Efectos Aleatorios en caso contrario. ${ }^{* * *}$, ${ }^{* *} \mathrm{y}^{*}$ supone el rechazo de la hipótesis nula al $1 \%, 5 \%$ y $10 \%$ respectivamente del nivel de significatividad.

El análisis estático de la rentabilidad se ha realizado mediante MCO encontrando Efectos Fijos en la muestra total y en las empresas brasileñas, no aporta resultados significativos aunque sean positivos de influencia del crecimiento sobre la rentabilidad. De otro modo, se encuentra influencias negativas del tamaño y el endeudamiento sobre la rentabilidad. De esta forma, según los resultados encontrados se puede decir que las empresas menos endeudadas y de menor tamaño son las más rentables.

En segundo lugar, los modelos dinámicos incluyen las variables dependientes retardadas por lo que también podemos contrastar la persistencia del crecimiento y de la rentabilidad. Los resultados obtenidos nos muestra que existe influencia negativa entre el crecimiento de dos periodos consecutivos, aunque no consideramos que exista persistencia ya que el valor de los coeficientes de la variable retardada no son próximos a la unidad. En el análisis de la rentabilidad encontramos influencia de la rentabilidad entre dos periodos consecutivos para la muestra general y para las empresas privadas brasileñas, pero al igual que en el caso del crecimiento, no podemos decir que sean persistentes debido a que el valor de los coeficientes no es próximo a 1. (Tabla 4).

Por otro lado, el análisis dinámico corroboran los resultados del estático sobre la influencia negativa de la rentabilidad sobre el crecimiento para el conjunto de la muestra y de las empresas de propiedad privada. Se mantiene la influencia positiva generalizada del tamaño sobre el crecimiento, lo que implica el rechazo de la ley del Efecto proporcional.

Tabla 4 - Análisis Dinámico de la Interrelación Crecimiento-Rentabilidad

\begin{tabular}{|c|c|c|c|c|c|c|c|c|c|}
\hline \multicolumn{5}{|c|}{ Análisis Dinámico del Crecimiento/GMM } & \multicolumn{5}{|c|}{ Análisis Dinámico de la Rentabilidad/GMM } \\
\hline & Total & Estatal & Brasileño & Extranjero & & Total & Estatal & Brasileño & Extranjero \\
\hline \multirow[t]{2}{*}{$\begin{array}{l}\text { Crec } \\
(-1)\end{array}$} & $-0,364^{* * *}$ & $-0,183^{* *}$ & $-0,380^{* * *}$ & $-0,366^{* * *}$ & Rent $(-1)$ & $0,063^{*}$ & $-0,082$ & $0,127^{* * *}$ & 0,023 \\
\hline & $(-19,54)$ & $(-2,55)$ & $(-17,04)$ & $(-16,81)$ & & $(1,68)$ & $(-0,97)$ & $(2,65)$ & $(0,44)$ \\
\hline \multirow[t]{2}{*}{$\begin{array}{c}\text { Rent } \\
(-1)\end{array}$} & $-0,114^{* * *}$ & $-0,020$ & $-0,082^{*}$ & $-0,151^{* * *}$ & Crec $(-1)$ & $-0,001$ & 0,001 & 0,002 & $-0,001$ \\
\hline & $(-3,31)$ & $(-0,70)$ & $(-1,94)$ & $(-4,97)$ & & $(-0,11)$ & $(0,36)$ & $(1,20)$ & $(-0,30)$ \\
\hline \multirow[t]{2}{*}{ Tamaño } & $142,691^{* * *}$ & $72,444^{* * *}$ & $132,025^{* * *}$ & $132,149^{* * *}$ & Tamaño & $-0,101$ & $-2,092$ & 0,443 & $-1,715$ \\
\hline & $(19,43)$ & $(8,42)$ & $(16,71)$ & $(20,97)$ & & $(-0,23)$ & $(-1,64)$ & $(0,87)$ & $(-1,65)$ \\
\hline \multirow[t]{2}{*}{ End } & 0,254 & 0,280 & 0,377 & 0,334 & End & $-0,096$ & $0,257^{*}$ & $-0,113$ & $-0,273^{*}$ \\
\hline & $(0,84)$ & $(0,48)$ & $(1,13)$ & $(0,76)$ & & $(-0,92)$ & $(1,95)$ & $(-0,98)$ & $(-1,71)$ \\
\hline \multirow[t]{2}{*}{ Liq } & $-0,442$ & 1,010 & 1,606 & $-11,899$ & Liq & 0,346 & 4,187 & 0,026 & 0,023 \\
\hline & $(-0,17)$ & $(0,12)$ & $(0,40)$ & $(-0,78)$ & & $(0,39)$ & $(1,24)$ & $(0,04)$ & $(0,02)$ \\
\hline $\mathrm{F}$ & $107,87^{* * *}$ & $17,31^{* * *}$ & $75,24^{* * *}$ & $57,02^{* * *}$ & $\mathrm{~F}$ & $5,32^{* * *}$ & $2,42^{* * *}$ & $5,49^{* * *}$ & $4,30^{* * *}$ \\
\hline $\mathrm{m}_{2}$ & $-2,10^{* *}$ & 0,23 & $-1,29$ & $-2,37^{* *}$ & $\mathrm{~m}_{2}$ & $-1,94^{*}$ & $-1,18$ & $-1,48$ & $-1,18$ \\
\hline Sargan & $2592,50^{* * *}$ & $281,51^{* * *}$ & $1931,95^{* * *}$ & $670,02^{* * *}$ & Sargan & $325,82^{* * *}$ & $242,55^{* * *}$ & $203,07^{* * *}$ & $178,70^{* * *}$ \\
\hline \multicolumn{10}{|c|}{$\begin{array}{l}\text { En la tabla se recogen los resultados de la estimación de los modelos dinámicos de crecimiento y rentabilidad estimados mediante el Método Generalizado de los } \\
\text { Momentos (GMM). Crecimiento (-1) y Rentabilidad (-1) son las variables retardadas un periodo. Se incluyen las variables de control Tamaño, Endeudamiento y } \\
\text { Liquidez.. En los modelos estimados mediante GMM se incorpora las variables dependientes retardadas, se muestra el test de buen ajuste del modelo mediante el } \\
\text { test F, el test de Arellano y Bond (1991) de no existencia de autocorrelación de segundo orden, expresado en ma, y el test de validez de los instrumentos } \\
\text { mediante el test de Sargan. }{ }^{* * *},{ }^{* *} \text { y }{ }^{*} \text { supone el rechazo de la hipótesis nula al } 1 \%, 5 \% \text { y } 10 \% \text { respectivamente del nivel de significatividad. }\end{array}$} \\
\hline
\end{tabular}




\section{Conclusiones}

Los aspectos claves para saber por qué unas empresas crecen más que otras son las hipótesis de ley del Efecto proporcional y de la persistencia de rentabilidad ya que son las bases para afrontar el estudio de la interrelación o no entre crecimiento y rentabilidad empresarial, desde un punto de vista empírico. Esto permite contrastar el comportamiento del crecimiento empresarial y conocer qué factores lo potencian.

Las empresas, empresarios e inversores han centrado su atención sobre las economías emergentes en busca de crecimiento y rentabilidad, seleccionando Brasil por su carácter de líder, tanto político como económico. De este modo, Brasil se ha convertido en unos de los principales destinos de las inversiones extrajeras directas por su altas tasas de crecimiento, de rentabilidades, debido principalmente a sus importantes reservas de recursos naturales y a su creciente demanda interna.

Los datos de panel y la estimación mediante Efectos Fijos y GMM nos han permitido estimar regresiones estáticas y dinámicas de datos de panel y solucionar problemas econométricos de heterogeneidad inobservable y el problema de endogeneidad, siendo más eficiente los resultados obtenidos.

Los resultados obtenidos en este trabajo se aproximan a los encontrados en el trabajo de Lee (2014) en el cual la tasa de rentabilidad del periodo anterior tiene un efecto negativo en la tasa de crecimiento empresarial del periodo siguiente, no encentrando relación significativa del crecimiento sobre la rentabilidad como el trabajo de Goddard et al. (2004).

Los principales resultados obtenidos nos indican que las empresas de Brasil que más crecen son las que obtienen menores tasas de rentabilidad. Esto se debe a la proceso de selección de proyectos, siendo elegidos primero los proyecto más rentables afrontando el resto de proyectos a pesar de ser menos rentables. De este modo, se encuentra también que las empresas que más crecieron en el periodo anterior son las que menos crecerán en el siguiente ya que los recursos utilizados en proyecto poco rentables no proporcionan los beneficios necesarios para reinvertirlos en la misma proporción.

Por otra parte, se rechaza la ley del Efecto proporcional ya que las empresas de mayor tamaño las que más crecen. Por lo tanto, el crecimiento de las empresas en Brasil se debe en mayor medida a factores de estructura de mercado como la concentración y el tamaño empresarial y no a factores de empresas basado sobre la eficiencia o la rentabilidad.

El trabajo aporta evidencias para establecer que el tipo de propiedad y control afecta sobre el comportamiento de la rentabilidad, ya que es donde se encuentra mayores diferencias. Se ha observado que las empresas de propiedad privada tienden a la persistencia de la rentabilidad, justificado por perseguir objetivos de rentabilidad para la satisfacción de los accionistas sus accionistas. Además un exceso de endeudamiento en las empresas privadas afecta negativamente a la rentabilidad en contra del efecto apalancamiento que tiene sobre las empresas públicas.

\section{Referencias}

Arellano, M., \& Bond, S., (1991): Some tests of specification for panel data: Monte Carlo evidence and an application to employment equations. The Review of Economic Studies, 58(2), 277-297.

Arnold, J.M., \& Jalles, J., (2014): Dividing the pie in Brazil: income distribution, social policies and the new middle class. IMF working paper, 1105.

Billett, M., King, T. \& Mauer, D., (2007): Growth opportunities and the choice of leverage, debt maturity, and covenants. The Journal of Finance, 62, 533-575.

Blundell, R., \& Bond, S., (1998): Initial conditions and moments restrictions in dynamic panel data models. Journal of Econometrics, 87, 115-143.

Broome, T., Robinson, J., \& Moore, W., (2009): Liquidity and the firm sizegrowth nexus Barbados. Economic Reviews, Working paper, Central Bank Barbados.

Coad, A., (2007): Testing the principle of growth of the fitter: the relationship between profits and firm growth. Structural Change and Economic Dynamics, 18(3), 370-386

Coad, A., (2009): The Growth of Firms: A Survey of Theories and Empirical Evidence. Edward Elgar Publishing.

Coad, A., Rao, R, \& Tamagni, F., (2011): Growth processes of Italian manufacturing firms. Structural Change and Economic Dynamics, 22(1), 54-70. Cowling, M., (2004): The growth-profit Nexus. Small Business Economics, 22(1), $1-9$.

García-Majón, J., \& Romero-Merino, M.E., (2012): Research, development, and firm growth. Empirical evidence from European top R\&D spending firms. Research Policy, 41(6), 1084-1092.

García-Ramos, R, \& García-Olalla, M., (2011): Estructura del consejo de administración en la empresa familiar vs no familiar: evidencia empírica en España. Revista Española de Financiacióny Contabilidad, 149, 35-64.

Gibrat, R. (1931). Les Inegalities Economiques. Paris: Sirey.

Goddard, J., McKillop, D.G., \& Wilson J.O.S. (2002): The Growth of US Credit Unions. Journal of Banking and Finance, 22, 2327-2356.

Goddard, J., Molyneux, P., \& Wilson, J., (2004): Dynamics of growth and profitability in banking.Journal of Money, Credit \& Banking, 36, 1069-1090.

Hsieh, M.F., \& Lee, C.C., (2010): The puzle between banking competition and profitability can be solved: international evidence from bank-level data. Journal of Financial Servies, 38, 135-157.

Jang, S., \& Park, K., (2011): Inter-relationship between firm growth and profitability. International Journal of Hospitality Management, 30(4), 10271035.

Lee, S., (2014): The relationship between growth and profit: evidence from firm-level panel data. Structural Change and Economic Dynamics, 28, 1-11.

López Vicente F. (2013): “La situación de la economía brasileña en el actual escenario económico mundial". Información Comercial Española, Revista de Economía, 875, 145-165.

Oliveira, B., \& Fortunato, A., (2006): Testing Gibrat's Law: empirical evidence from a panel of Portuguese manufacturing firms. International Journal of the Economics of Business, 13(1), 65-81.

Oliveira, B., \& Fortunato, A. (2008): The dynamics of the growth of firms: evidence from the services sector. Empirica, 35(3), 293-312.

Orgaz L., Molina L. y Carrasco C. (2011): El creciente peso de las economías emergentes en la economía y gobernanza mundiales. Los países BRIC. Documentos ocasionales no 1101, Banco de España.

Pettinger, R, (2013): Strategy, investment, behaviour and results. Tourism \&Management Studies, 9(1), 49-57.

Roodman, D., (2009): How to do xtabond2: an introduction to difference and system GMM in Stata. Stata Journal StataCorp LP, 9.

Serena Garralda J.M. (2011): La economía de Brasil desde los 90. Una historía de éxitos y retos pendientes. Boletín de Estudios Económicos, 66(204), 483510).

Suárez Suárez A.S. (2014): Decisiones óptimas de inversión y financiación en la empresa. Pirámide, Madrid.

Sutton J. (1997): Gibrat's legacy. Journal of Economic Literature, 35(1), 40-59.

Wagner, J., (1992): Firm Size, Firm Growth and Persistence of Chance? Testing GIBRAT's Law with Establishment Data from Lower Saxny, 1978-1989. Small Business Economics, 4(2), 125-131.

Recibido: 12.06.2014 Aceptado: 22.03.2015 